\title{
Estrés laboral y su relación con las condiciones de trabajo
}

\author{
Reyna Jarelis Suárez Duarte \\ Lismarck Yohanny Campos Sequeira ${ }^{2}$ \\ Jenny del Socorro Villanueva ${ }^{3}$ \\ Concepción Mendoza Castro ${ }^{4}$
}

\section{INFORMACIÓN DEL ARTÍCULO}

\author{
Recibido: 30.04 .2020
}

Aprobado: 30.05 .2020

\section{Palabras claves:}

Ambiente de trabajo, Condiciones de trabajo, Estrés Laboral, Ruido.

\section{RESUMEN}

El trabajo en hospitales o centros de salud ha sido considerado como una fuente estresante, porque implica estar continuamente en contacto con el dolor y a menudo con la muerte. Las cargas de trabajo, las jornadas laborales extensas y el ambiente laboral, son factores que contribuyen en el estado anímico de los colaboradores del Centro de Salud Adán Barillas Huete del municipio de Juigalpa, departamento de Chontales. El propósito es determinar el estrés laboral y su relación con las condiciones laborales de los colaboradores durante el año 2019. El tipo de investigación desarrollada fue concluyente explicativa, de naturaleza mixta, con un muestreo probabilístico estratificado conformado por 20 colaboradores, para lo cual se utilizaron la entrevista y encuesta. Los resultados evidencian que el 55\% siempre y casi siempre han experimentado estrés y $45 \%$ algunas veces, Los síntomas del estrés en un 95\% son de índole fisiológica siendo la jaqueca y dolores de cabeza el principal síntoma, condiciones labores como la limpieza del área, iluminación y el ruido no son causas para el estrés laboral. Concluyendo que con los resultados de la aplicación de la técnica del chi cuadrado de Pearson se respalda la aceptación de la hipótesis nula, reafirmando que las condiciones laborales no influyen en el estrés laboral de los colaboradores, dado que el resultado de cada asíntota bilateral fue mayor a 0.05.

\section{Labor stress and its relationship with working conditions}

\section{ABSTRACT}

Working at hospitals or health centers has been considered a stressful job, because it implies being in constant contact with pain and death. Workloads, extensive working hours and the work environment are contributing factors for the employees' mood at Adan Barilla Huete Health Center

\footnotetext{
${ }^{1}$ Licenciada en Administración de Empresas. Profesora de la Universidad Nacional Autónoma de Nicaragua, Managua Facultad Regional Muldisciplinaria - Chontales. Email: jaresuadu13@gmail.com

2 Licenciada en Administración de Empresas. Profesora de la Universidad Nacional Autónoma de Nicaragua, Managua Facultad Regional Muldisciplinaria - Chontales. Email: lismarckc15@gmail.com

3 Doctora en Ciencias Económicas. Profesora de la Universidad Nacional Autónoma de Nicaragua, Managua - Facultad Regional Muldisciplinaria - Chontales. Email: jennyvillanueva7o@yahoo.es (1): https://orcid.org/oooo-ooo1-8151-1061

4 MÁster en Administración Funcional de Empresas e Instituciones. Profesora de la Universidad Nacional Autónoma de Nicaragua, Managua - Facultad Regional Muldisciplinaria - Chontales. Email: cony2omend@yahoo.es 이: https://orcid. org/oooo-0003-3226-0715
} 
in Juigalpa city. The purpose is to determine work stress and its relationship with the working conditions of employees during the 2019. The type of research was conclusive explanatory, of a mixed nature, with a probabilistic sampling made up of 20 collaborators, it was used the interview and survey. The results show that $55 \%$ have always and almost always experienced stress and $45 \%$ sometimes. The symptoms of stress in $95 \%$ are physiological, it was mostly provoked by headaches, working conditions such as cleaning the area, lighting and noise are not causes for work stress. Concluding that, the acceptance of the null hypothesis is supported with the results of the application of Pearson's chi-square technique, claiming that working conditions do not influence the work stress of employees.

\section{Introducción}

Actualmente el estrés es uno de los causantes de numerosas enfermedades, tanto físicas como mentales, debido a las condiciones laborales, sociales, personales y económicas a las que nos enfrentamos cotidianamente, en las cuales se hacen presente diferentes eventos que pueden considerarse estresantes. Por ello es importante saber identificar adecuadamente la presencia de estrés. En el estrés laboral existen desencadenantes que están ligados específicamente al desempeño de una profesión. El trabajo en hospitales o centros de salud ha sido considerado como una fuente estresante, porque implica estar continuamente en contacto con el dolor y a menudo con la muerte, además de las cargas de trabajo y las tensiones a las que se somete el colaborador.

En el centro de salud Adán Barillas Huete, últimamente el exceso de trabajo y las largas jornadas laborales está causando improductividad en el trabajo y problemas de salud, ya que los colaboradores frecuentemente se despiertan preocupados y sienten el peso de volver a su lugar de trabajo, es notorio que padecen de enfermedades causadas por el estrés. Se ha identificado la existencia de algunos factores que están causando estrés laboral en los colaboradores de esta institución.

En relación con el personal administrativo, el volumen de trabajo resulta demasiado cansado, ya que la mayoría del tiempo tiene que sacrificar sus horas de descanso para cumplir con sus tareas asignadas. En ocasiones son días enteros de trabajo, porque las responsabilidades son muchas y hay poco personal de apoyo administrativo laborando, lo que también conlleva a una acumulación de trabajo, encontrándose constantemente preocupados, cansados y ansiosos.

Otro causante del estrés es el horario de trabajo, que varía en dependencia del puesto, el personal administrativo tiene determinado en su contrato trabajar ocho horas diarias, sin embargo, por el volumen de trabajo esto no es suficiente, por ello tienen que permanecer largas horas en sus oficinas hasta dar por terminada sus obligaciones. De igual manera, el personal de salud médico y enfermeros tiene que cumplir con su trabajo que implica el contacto a diario con el usuario en ocasiones lidiando con diferentes temperamentos, agresiones verbales, quejas por parte del paciente y con la exposición a riesgo y al contagio de enfermedades. Así mismo sus jornadas 
extraordinarias que desempeñan implican sacrificios de horario de alimentación, ocio, descanso y sueño

¿Acaso esto ocasiona que los colaboradores resulten cansados al final del día, para poder disfrutar de actividades recreativas en la noche?

En relación con el entorno laboral, muchos de los colaboradores además de estar constantemente con un ritmo de trabajo agitado, en el centro de salud no se brindan las condiciones necesarias porque desde que fue fundado no ha tenido ningún cambio en su infraestructura a pesar de que el número de colaboradores ha ido aumentando, también es evidente que sus actividades las realizan en ambientes inseguros, expuestos a accidentes, las oficinas administrativas y de recursos humanos llenas de cables eléctricos e interruptores que pueden provocar algún cortocircuito, de igual manera los doctores se encuentran en cubículos compartidos para dar consulta médica a los pacientes.

Las circunstancias anteriores pueden llevar a la institución a disminuir la calidad del servicio y provocar ausentismo ya que las personas con mucho ritmo laboral, con un entorno conflictivo y un ambiente de trabajo inadecuado son menos productivas.

El estudio presentado por Borjan, (2016) Estrés laboral en personal de enfermería en la unidad de cuidados intensivos pediátricos Hospital Escuela Universitario Tegucigalpa-Hondura enero-marzo 2016, determinó el nivel de estrés laboral en el personal de enfermería y abordó sobre las manifestaciones de estrés del personal, nivel de agotamiento emocional y despersonalización y niveles de realización personal concluyendo que las manifestaciones presentadas en los colaboradores son de carácter fisiológico, cognoscitivo, emocional y de conducta, entre los más comunes: Fatiga, dolor de cabeza, aceleración del ritmo cardiaco, sudoración excesiva, nauseas, vómitos, alteraciones visuales, concentración deficiente, dificultad para identificar problemas, ansiedad, depresión, entre otros.

Así mismo Alaniz, (2016) en su estudio abordo el "Estrés Laboral en médicos residentes de los hospitales públicos de Managua abril a mayo 2016". Teniendo como objetivo analizar los factores asociados al estrés laboral en los médicos residentes de los hospitales escuelas públicos, concluye que los principales factores asociados al estrés laboral de los medicos residentes fueron la carga laboral (volumen de pacientes) y las relaciones interpersonales.

El estrés organizacional ha sido identificado como una de las problemáticas de importancia en los últimos tiempos, que no solo afecta a la persona como tal, sino también el ambiente que lo rodea, por ende, es importante estudiar esta temática ya que en la actualidad es un problema común en las organizaciones y por ello es 
necesario conocer la fuente que lo provoca, las causas específicas y las consecuencias de una situación que no es atendida de forma crucial.

El objetivo general se enfoco en determinar el estrés laboral y su relación con las condiciones laborales de los colaboradores del centro salud Adán Barillas Huete en el municipio de Juigalpa, Chontales.

\section{Literatura}

Se entiende por estrés laboral, como el conjunto de fenómenos o agentes estresantes derivados directamente del trabajo. En un estado patológico crónico ocasionado por las condiciones habituales de trabajo en las que se encuentra una persona. Afecta a su cuerpo y a su mente. Y repercute, por supuesto, no solo sobre su bienestar personal, sino también sobre su rendimiento (Acosta, 2008, p. 36). De igual manera, Unión Nacional de Trabajadores [UGT] Andalucía (2009) señala que: el proceso en el que las demandas ambientales comprometen o supera la capacidad adaptativa de un organismo, dando lugar a cambios biológicos y psicológicos que pueden situar a la persona en riesgo de enfermedad. Definido como el estado de fatiga física y psicológica de un individuo provocado por el exceso de trabajo, de desórdenes emocionales, ansiedad y miedo (p. 27).

Los efectos del estrés se inician en el modelo de Hans Selye, teórico pionero del estrés. Este modelo, el síndrome de adaptación general (SAG), señala que ocurre el mismo conjunto de reacciones fisiológicas al estrés sin importar su causa particular. Feldman (2006) expresa que el modelo tiene tres fases: la primera etapa de alarma y movilización ocurre cuando se cobra conciencia de la presencia de un estresor (...) Sin embargo, si persiste el estresor se pasa a la siguiente etapa del modelo. En la etapa de resistencia, el individuo se prepara para combatir el estresor (...) Si la resistencia es inadecuada, la persona entra en la última etapa del modelo, la de agotamiento. Durante esta etapa, la capacidad para adaptarse al estresor disminuye a tal grado que aparecen las consecuencias negativas del estrés como la afección física y los síntomas psicológicos (p. 427).

Son muchas las causas que pueden generar estrés en el ambiente laboral, tales como excesiva demanda de trabajo, presión por los tiempos entre cada proceso, lograr las metas propuestas. "El estrés laboral aparece cuando por la intensidad de las demandas laborales o por problemas de índole organizacional, el trabajador empieza a experimentar vivencias negativas asociadas al contexto laboral" (Rodriguez, Molerio, \& Roque, 2002, p. 10).

Volumen de trabajo. El estrés relacionado con el trabajo es la reacción negativa que se produce cuando las exigencias del trabajo superan la capacidad para hacerles frente. Esto significa que la carga de trabajo se define como el "conjunto de obligaciones 
psicofísicas a los que se ve sometido el trabajador a lo largo de su jornada laboral" (UGT, sf, pág.10). La consecuencia de una excesiva carga de trabajo es la fatiga que podemos definirla como la disminución de la capacidad física y mental de un trabajador después de haber realizado una actividad durante un periodo de tiempo. Las cargas laborales se clasifican de la siguiente manera: carga física y carga mental.

Ritmo de trabajo. Son exigencias temporales ligadas a la carga de trabajo. Es el tiempo necesario para realizar una determinada tarea. Para evaluarlo no solo tenemos en cuenta el factor tiempo sino también debemos tener en cuenta el nivel de concentración y atención necesaria para la ejecución de las tareas, la rapidez con las que hay que realizar esas tareas, si tienen plazos cortos o estrictos, o determinados por máquinas, clientes, procesos productivos, etc.

Horario de trabajo. La Asamblea Nacional de Nicaragua (1996) en el artículo 29 de la Ley 185 Código del Trabajo, e entiende por jornada de trabajo: el tiempo durante el cual el trabajador se encuentra a disposición del empleador, cumpliendo sus obligaciones laborales. Se considera que el trabajador se encuentra a disposición del empleador desde el momento en que llega al lugar donde debe efectuar su trabajo, o donde recibe órdenes o instrucciones respecto al trabajo que se ha de efectuar en la jornada de cada día, hasta que pueda disponer libremente de su tiempo y de su actividad. De igual forma, en el artículo 50, se establece que el día natural para los efectos del trabajo es el comprendido entre las seis de la mañana y las ocho de la noche. Trabajo nocturno es el que se presta entre las ocho de la noche y las seis de la mañana del día siguiente.

Los efectos del estrés son aspectos que juega un papel esencial y es prácticamente el núcleo principal del estrés es la ansiedad. Siempre que haya estrés aparecerá la ansiedad. La UGT de Andalucía (2009) afirma que la ansiedad es una manifestación afectiva que se caracteriza por el temor ante algo inconcreto y difuso, carente de objeto exterior, en la que el individuo tiene una impresión de indefensión. Esta ansiedad va a provocar una gran alteración de toda la psicología del sujeto (p. 31). Desde otra perspectiva, los síntomas del estrés sobre el funcionamiento del organismo son de índole fisiológica, psicológica y conductual. (Mutua Colaboradora con la Seguridad Social, 2008).

El estrés y condiciones laborales, dentro del origen potencial de estrés, existen tres categorías causantes potenciales: Ambientales, organizacionales e individuales. Al hablar de "Las condiciones ambientales del trabajo nos referimos a las circunstancias físicas que rodean al empleado como ocupante de un puesto en la organización, es decir, al ambiente físico que rodea al empleado mientras desempeña un puesto" (Chiavenato, 2007, p. 334).

Es importante conocer las condiciones que causan estrés, algunos de ellos pueden surgir directamente de lo que las personas experimentan en el trabajo. "Así como la 
incertidumbre del ambiente influye en el diseño de la estructura de una organización, también ejerce influencia en los niveles del estrés en los empleados" (Robbins, 2004, p. 90). La incertidumbre tecnológica es un factor ambiental que produce estrés.

Iluminación. Es la acción o efecto de iluminar. En la técnica se refiere al conjunto de dispositivos que se instalan para producir ciertos efectos luminosos, tanto prácticos como decorativos. Según Chiavenato (2007) afirma que es la cantidad de luz que incide en el lugar de trabajo empleado. No se trata de la iluminacion general, si no de la cantidad de luz en el punto focal del trabajo. Asi, los estándares de la iluminación se establecen de acuerdo con el tipo de tarea visual que el empleado debe realizar (p. 334).

Ruido. Es considerado como un sonido o barullo indeseable. En lo que respecta, el sonido tiene dos carácteristicas principales: la frecuencia e intensidad. La frecuencia es el número de vibraciones por segundo emitidas por la fuente del ruido , y se mide en ciclos por segundo (cps). La intensidad del sonido se mide en decibeles (db). Las investigaciones realizadas muestran que el ruido no provoca disminución en el desempeño del trabajo,sin embargo, la influencia del ruido perjudica en gran cantidad la salud del empleado y principalmente su audición. (Chiavenato,2007, p. 334)

Temperatura. Apoyado en Asamblea Nacional de Nicaragua (2007) en la Ley $\mathrm{N}^{\circ}$ 618, Ley general de higiene y seguridad laboral en el trabajo en el articulo 210, establece "Que se deben instalar termómetros para la comprobación de la temperatura de todos los equipos y accesorios que contengan fluidos deben contar con estos instrumentos de medición para llevar el control de la temperatura seleccionada”.

En lo que respecto con Chiavenato (2007) afirma que una de las condiciones ambientales importantes es la temperatura. Existen puestos cuyo lugar de trabajo se caracteriza por elevadas temperaturas como es el caso de la proximidad a los hornos en una siderúrgica de una empresa de cerámica, o de una herreria etc, en los cuales los ocupantes necesitan vestir ropa adecuada para proteger su salud. En el otro extremo hay puestos cuyo lugar de trabajo impone temperaturas muy bajas, como en los casos de frigorificos que exigen ropa adecuada para la protección. (p.335).

Humedad. Es una medida del vapor de agua que contiene el aire, para Chiavenato (2007): es consecuencia del alto contenido higrométrico del aire. Existen condiciones ambientales de gran humedad en el lugar de trabajo, es el caso de la mayor parte de las fabricas textiles, que exigen un elevado grado higrométrico para el tratamiento de los hilos, por otra parte, existen condiciones ambientales de poca o nula presencia de la humedad; por ejemplo, la industria de la cerámica donde el aire debe ser seco (p.336).

Consecuentemente, el trabajo puede ser estresante y las demandas del empleo pueden alterar el equilibrio trabajo-vida de las personas. Robbins (2004) Clasifica los factores organizacionales de la siguiente manera: las exigencias de las tareas abarcan 
el diseño de puesto (...) condiciones laborales y disposición física del trabajo. Las exigencias del rol, se relaciona con las presiones que se imponen a una persona por el rol que cumple en la organización (...) las exigencias interpersonales referidas a las presiones que ejercen otros empleados, la estructura de la organización, que define el grado de las reglas y normas, así como también el liderazgo organizacional, que representa el estilo gerencial de los altos ejecutivos de la organización. Y por último la etapa de la vida de la organización, las etapas de fundación y declinación son particularmente estresante (p. 579).

Los factores individuales, abarca los factores de la vida privada de los empleados. Principalmente, estos factores son los asuntos familiares, problemas económicos, personales y características propias de la personalidad" (Robbins, 2004, p. 580). La persona promedio trabaja de 40 a 50 horas por semana, pero las experiencias y dificultades por las que pasan en las otras 120 o más horas que tiene la semana pueden verter en el trabajo.

Por otra parte, según Whetten, Kim, \& Cameron (2011), la mejor manera para manejar el estrés es eliminar o minimizar los factores que lo producen por medio de las estrategias: Estrategias de ejecución; estas crean o establecen un nuevo entorno para el individuo que no contiene factores de estrés. El segundo método más eficaz es que los individuos mejoren su capacidad general de manejar el estrés al incrementar su elasticidad personal. Estas se llaman estrategias proactivas y están diseñadas para iniciar una acción que resista los efectos negativos del estrés. Finalmente, es necesario desarrollar técnicas a corto plazo para manejar los factores de estrés cuando se requiere una respuesta inmediata. Estas estrategias son reactivas; son aplicadas como remedio en el momento para reducir los efectos temporales del estrés (p.116).

\section{Metodología de investigación}

En lo referente al diseño metodológico, se clasificó según su alcance como una investigación concluyente, de tipo explicativa, ya que se describieron las causas y efectos de la relación entre las variables del estrés y condiciones laborales. Bernal (2006) afirma que la investigación explicativa tiene como fundamento la prueba de hipótesis y busca que las conclusiones lleven a la formulación o el contraste de leyes o principios científicos. Cuando en una investigación, el investigador se plantea como objetivos estudiar el porqué de las cosas, los hechos, fenómenos o situaciones, a estas investigaciones se les denomina explicativas (p. 115).

Ahora bien, según el carácter de la medida la investigación se enfoco en cuantitativa y cualitativa. Según el tipo de investigación se inició con una investigación documental con la recopilación y selección de información a través de la lectura de libros y documentos de comportamiento organizacional, estrés y psicología. Según Tamayo (2008) "la investigación documental es la que se realiza con base en la revisión 
de documentos, manuales, revistas, periódicos, actas científicas, conclusiones de simposios y seminarios y/o cualquier tipo de publicidad considerado como fuente de información" (p. 84). Y se finalizó con una investigación de campo a través de la aplicación de una entrevista al responsable del área de recursos humanos y encuestas a demás colaboradores de la institución. Tamayo (2008) Considera que una investigación "Es de campo cuando los datos se recogen directamente de la realidad, por lo cual los denominamos primarios, su valor radica en que permiten cerciorarse de las verdaderas condiciones en que se han obtenido los datos" (p. 83).

Según el alcance temporal fue de diseño transversal, porque se recopilaron los datos durante el año 2019. La población que labora en el centro de salud está conformada por 128 colaboradores, sin embargo, se aplicó un muestreo probabilístico estratificado. Según Tamayo (2008) en este tipo de muestra se divide la población en estratos de acuerdo con ciertas características de las unidades de población, y luego se extrae al azar un determinado número de unidades proporcionales a cada estrato de acuerdo con la población total que representa la población (p.101).

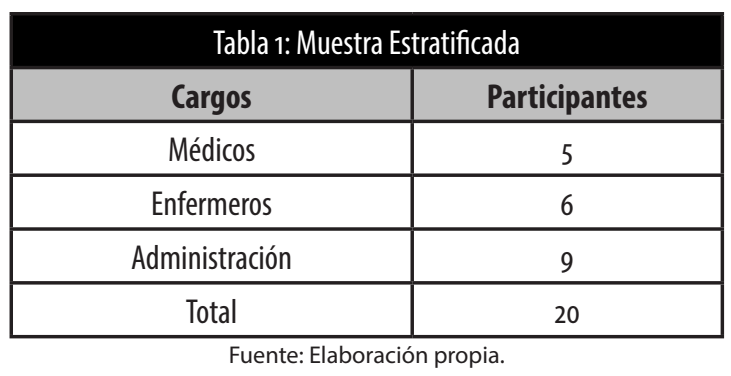

El instrumento utilizado fue la encuesta con un total de 10 preguntas con una escala Likert de siempre, casi siempre, algunas veces y nunca, además la entrevista con un total de 5 interrogantes. Para la validez y consistencia de las preguntas del instrumento encuesta, se realizó un pilotaje a 3 colaboradores del Centro de Salud Adán Barillas Huete. En lo que respecta al procesamiento de la información tanto para la encuesta como la técnica, se utilizó el software SPSS versión 21. posteriormente a través del software SPSS y Alpha de Cronbach se midió la fiabilidad de la información, dando como resultado o.8 que significa que es bueno. Soto \& Lautenschlager (2003) Aseveran que: "Esta prueba estadística se dirige a constatar el coeficiente Alpha obtenido en la muestra frente a un valor poblacional respectivo o hipotizado" (p.128).

Además, para establecer la relación con las condiciones laborales y el estrés laboral se utilizó la técnica de Chi-Cuadrado para comprobar la hipótesis planteada.

1. Ho: Las condiciones laborales no influyen en el estrés laboral de los Colaboradores del centro de salud "Adán Barillas Huete" 


\section{INVESTIGACIÓN EDUCATIVA}

2. H1: Las condiciones laborales influyen en el estrés laboral de los colaboradores del centro de salud "Adán Barillas Huete"

\section{Resultados}

En esta investigación participaron 20 hombres y mujeres con un $75 \%$ del personal encuestado del centro de salud son del sexo femenino. En la figura 1, se muestra que:

\section{Figura 1: Implica tu trabajo una alta responsabilidad}

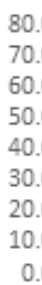

80.0
70.0
60.0
50.0
40.0
30.0
20.0
10.0
0.0

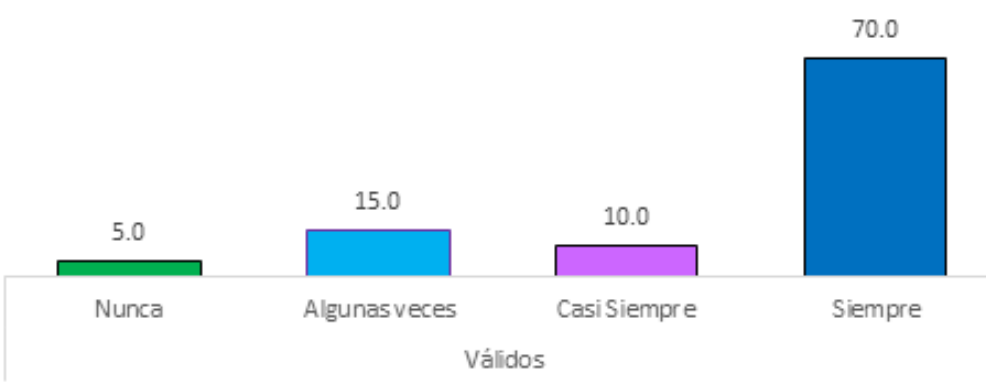

Los resultados demuestran que un 55\%, el personal encuestado siempre y casi siempre se encuentra estresado, sin perder de vista que el otro 45 algunas veces se han sentido estresado.

Figura 2: Te sientes frecuentemente estresado

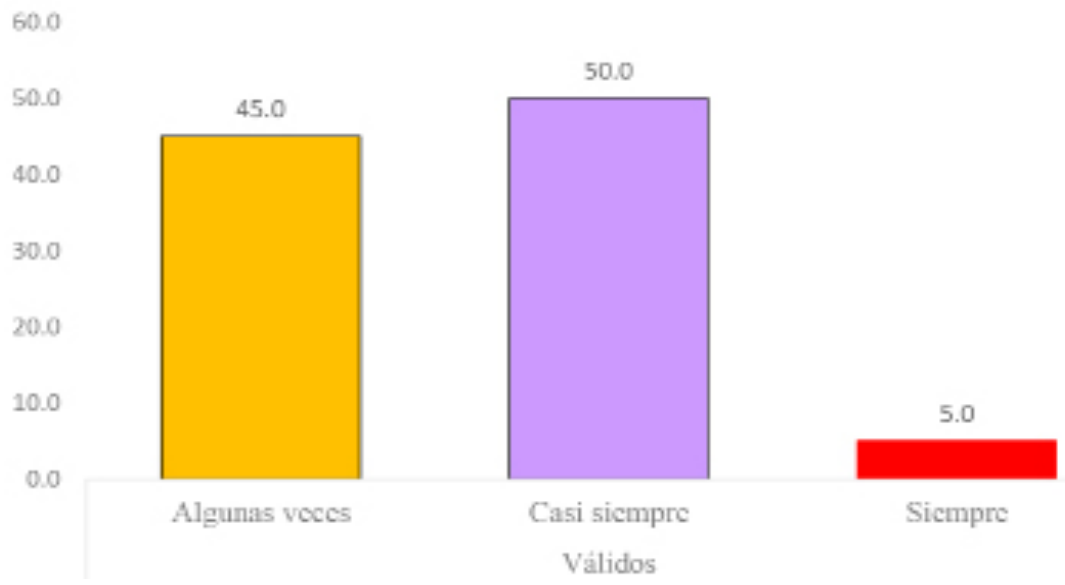


Es evidente que la mayoría de los colaboradores tienen un alto grado de responsabilidad en el cargo que ocupan visualizándose que el $80 \%$ siempre y casi siempre implica una alta responsabilidad. Sin embargo desde el punto de vista del responsable de recursos humanos, todas las áreas asumen responsabilidades aunque unas más que otras. Además el nivel de exigencias es alto en el caso del personal administrativo todo tiene fecha estipulada para entrega y el personal médico además de realizar su trabajo y turnos tiene que cumplir con un plan de metas mensualmente establecidas por Ministerio de Salud.

Figura 3: Te sientes presionado por parte de personas de un cargo superior

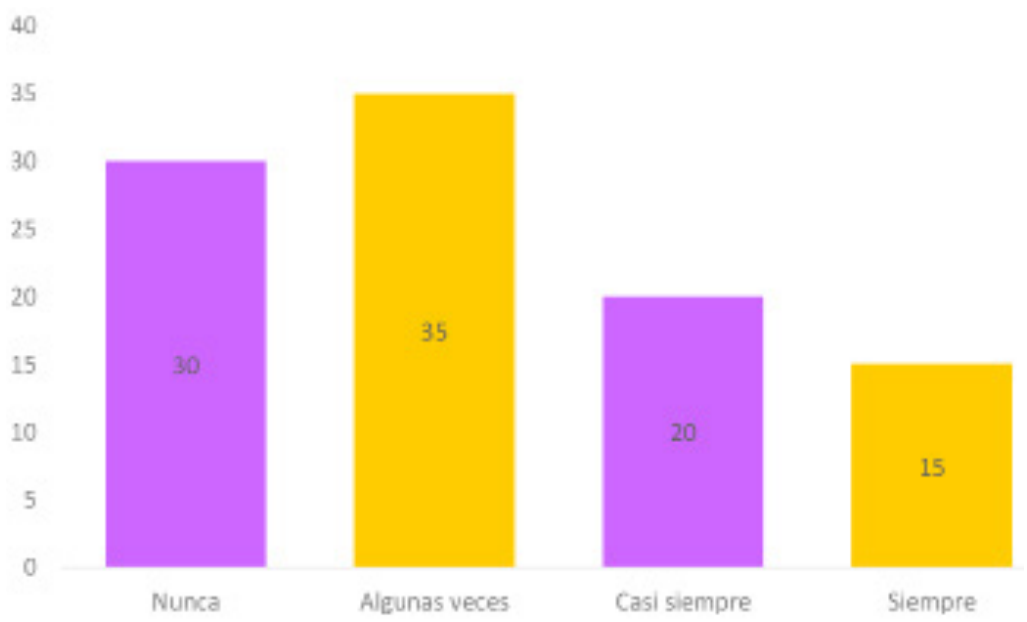

Notoriamente, los colaboradores del centro de salud confirmaron sentirse presionado por parte de personas con un cargo superior, ya que los resultados reflejan que en promedio el $70 \%$ siempre, casi siempre y algunas veces están presionados, esto debido a al ritmo de trabajo intenso en la institución. El responsable de recursos humanos enfatizo que la presión de trabajo recae más en los doctores y enfermeras, siempre están trabajando bajo presión, sumado al cansancio de los turnos que realizan. 
Figura 4: Tienes jornadas laborales muy extensas

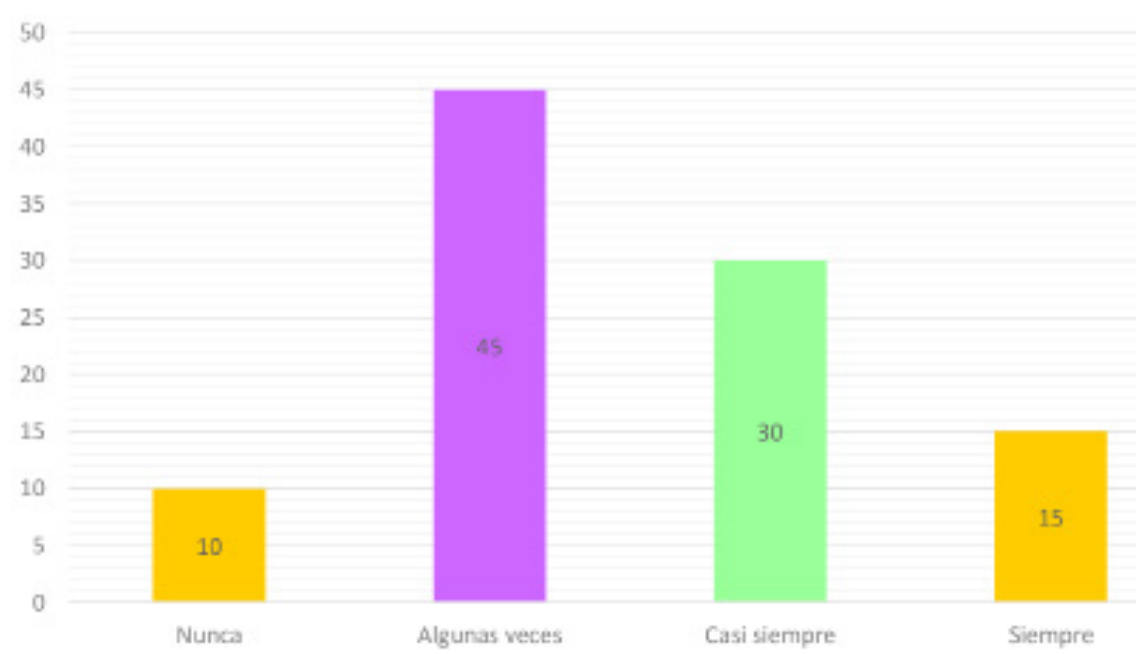

Los resultados expresan que el 90\% de los colaboradores algunas veces, siempre y casi siempre sus jornadas laborales son muy extensas.

Figura 5: Sientes jaquecas o dolores de cabeza

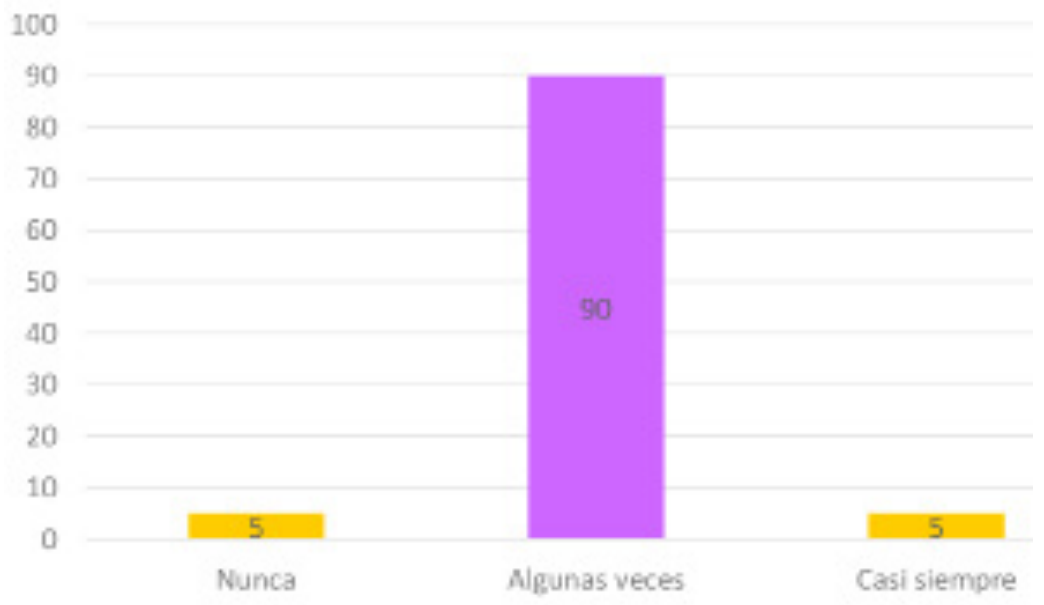

Referente a los síntomas del estrés sobre el funcionamiento del organismo son de índole fisiológica, psicológica y conductual. Es importante destacar que lo síntomas que más están afectando a los colaboradores son los físicos en especial los constantes 
dolores de cabeza en un 95\%. En lo que respecta con la relación de las condiciones laborales y su implicación con el estrés laboral se encontró:

Tabla 2: Relación de las instalaciones del área y el estrés laboral

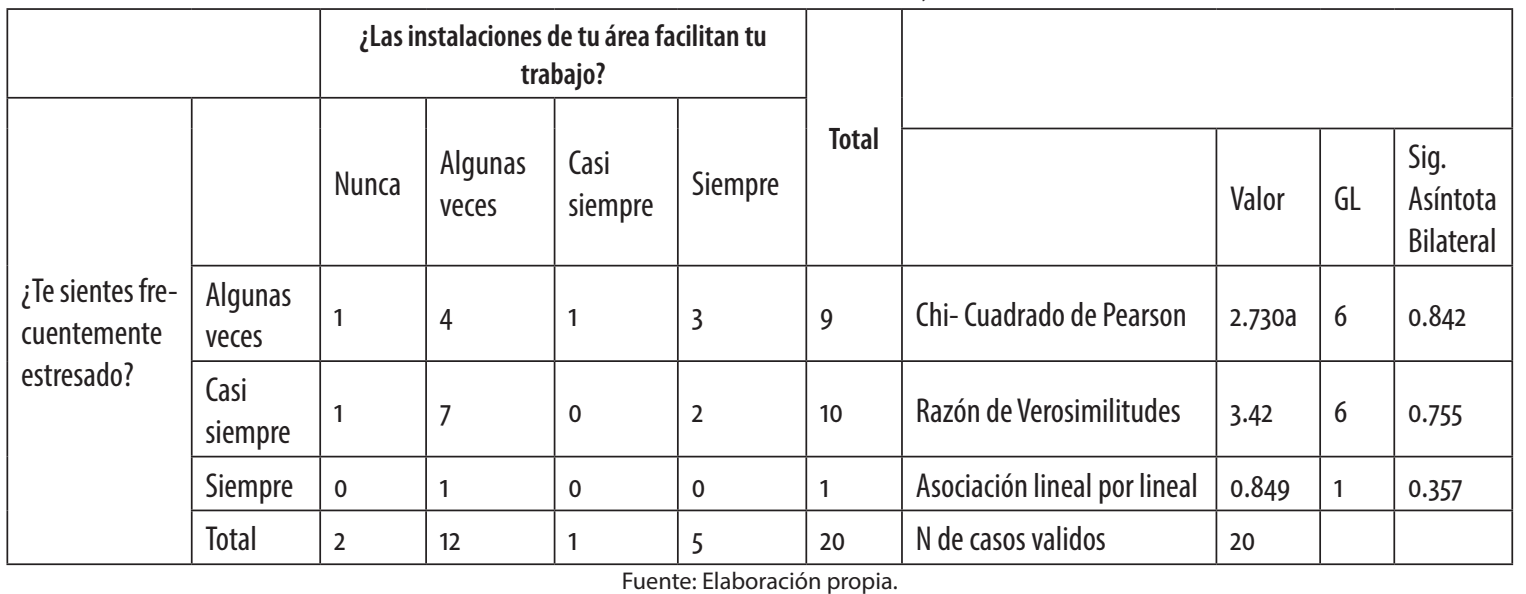

Es importante destacar que los colaboradores realmente en un $100 \%$ han estado estresados en algún momento, esto se refleja en los 20 colaboradores. El 90\% representado por 18 colaboradores, evidencian que las instalaciones de las áreas del centro de salud les facilitan el desarrollo de su trabajo. Al aplicar la técnica de chi cuadrado a ambas variables dio como resultado una significancia asíntota bilateral de 0.842

Tabla 3: Relación entre la limpieza y el estrés laboral

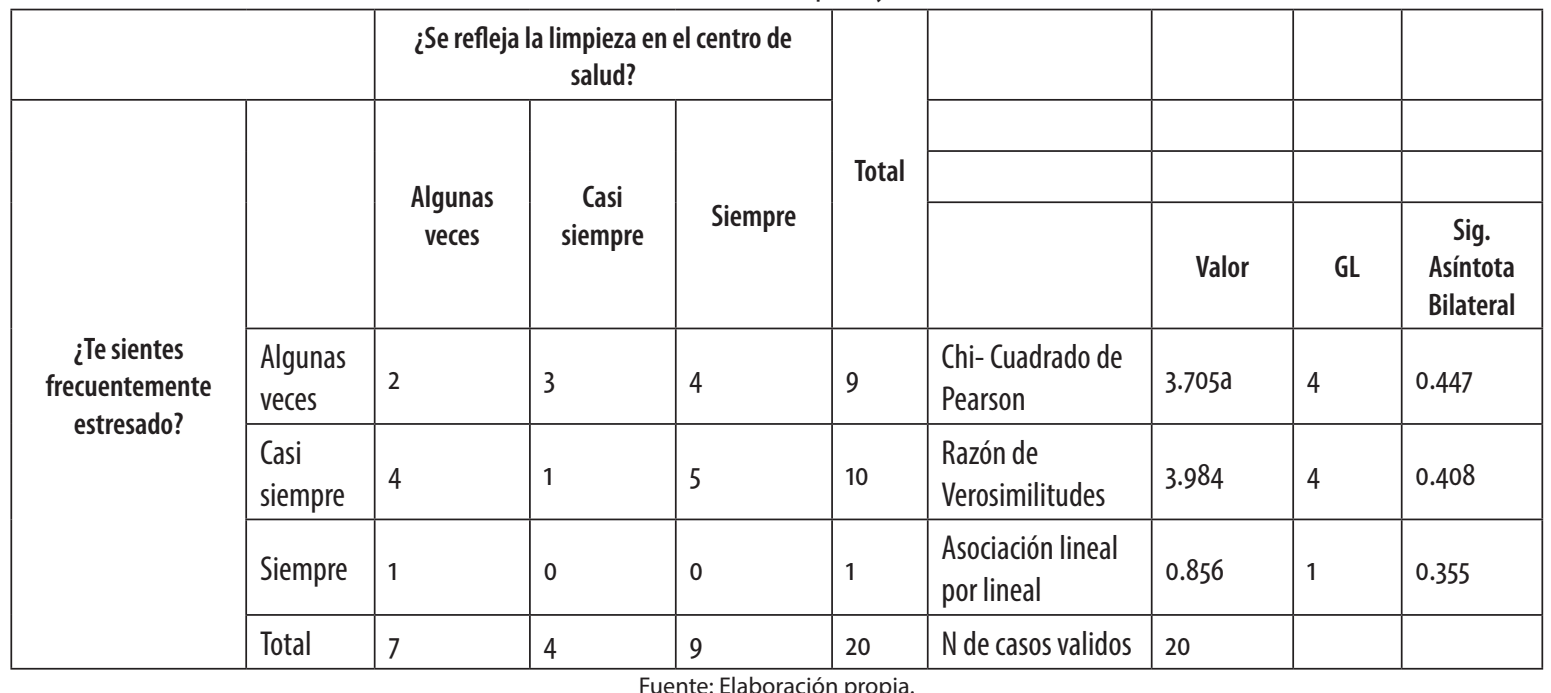


Es trascendental subrayar que los colaboradores realmente en un $100 \%$ han estado estresados en algún momento, esto se refleja en los 20 colaboradores al relacionarlo con la limpieza del centro de salud, 13 colaboradores que representa el 65\% expresaron que siempre y casi siempre se realiza limpieza de cada una de las áreas. $\mathrm{Al}$ aplicar la técnica de chi cuadrado a ambas variables dio como resultado una significancia asíntota bilateral de 0.447 .

Tabla 4: Relación de la iluminación y el estrés laboral

\begin{tabular}{|c|c|c|c|c|c|c|c|c|c|c|}
\hline & & \multicolumn{4}{|c|}{$\begin{array}{l}\text { ¿La iluminación de tu área de trabajo es } \\
\text { adecuada? }\end{array}$} & \multirow{3}{*}{ Total } & & & & \\
\hline \multirow{6}{*}{$\begin{array}{l}\text { ¿Te sientes } \\
\text { frecuentemente } \\
\text { estresado? }\end{array}$} & & \multirow{2}{*}{ Nunca } & \multirow{2}{*}{$\begin{array}{l}\text { Algunas } \\
\text { veces }\end{array}$} & \multirow{2}{*}{$\begin{array}{l}\text { Casi } \\
\text { siempre }\end{array}$} & \multirow{2}{*}{ Siempre } & & & & & \\
\hline & & & & & & & & Valor & GL & $\begin{array}{c}\text { Sig. } \\
\text { Asíntota } \\
\text { Bilateral }\end{array}$ \\
\hline & $\begin{array}{l}\text { Algunas } \\
\text { veces }\end{array}$ & 1 & 2 & 0 & 6 & 9 & $\begin{array}{l}\text { Chi-Cuadrado de } \\
\text { Pearson }\end{array}$ & $5.283 a$ & 6 & 0.508 \\
\hline & $\begin{array}{l}\text { Casi } \\
\text { siempre }\end{array}$ & 0 & 5 & 1 & 4 & 10 & $\begin{array}{l}\text { Razón de } \\
\text { Verosimilitudes }\end{array}$ & 6.363 & 6 & 0.384 \\
\hline & Siempre & 0 & 1 & 0 & 0 & 1 & $\begin{array}{l}\text { Asociación lineal por } \\
\text { lineal }\end{array}$ & 1.143 & 1 & 0.285 \\
\hline & Total & 1 & 8 & 1 & 10 & 20 & $\mathrm{~N}$ de casos validos & 20 & & \\
\hline
\end{tabular}

Físicamente en un $100 \%$ los colaboradores se sintieron estresados, al relacionar con la iluminación del centro de salud se observa en un $55 \%$ equivalente a 11 colaboradores siempre y casi siempre la iluminación de su área de trabajo es adecuada. Al aplicar la técnica de chi cuadrado a ambas variables dio como resultado una significancia asíntota bilateral de 0.508 . 
Tabla 5: Relación del ruido y el estrés laboral

\begin{tabular}{|c|c|c|c|c|c|c|c|c|c|c|}
\hline & & & $\begin{array}{r}\text { demasiado } \\
\text { desarro }\end{array}$ & $\begin{array}{l}\text { uido en el ár } \\
\text { is tu trabajo }\end{array}$ & en que & & & & & \\
\hline & & & Alqunas & Casi & & & & & & \\
\hline & & Nunca & veces & siempre & Siempre & & & Valor & GL & $\begin{array}{c}\text { Sig. } \\
\text { Asíntota } \\
\text { Bilateral }\end{array}$ \\
\hline $\begin{array}{c}\begin{array}{c}\text { ¿Te sientes } \\
\text { frecuentemente }\end{array}\end{array}$ & $\begin{array}{l}\text { Algunas } \\
\text { veces }\end{array}$ & 2 & 0 & 1 & 6 & 9 & $\begin{array}{l}\text { Chi- Cuadrado } \\
\text { de Pearson }\end{array}$ & $7.878 \mathrm{a}$ & 6 & 0.247 \\
\hline estresado? & $\begin{array}{l}\text { Casi } \\
\text { siempre }\end{array}$ & 3 & 3 & 1 & 3 & 10 & $\begin{array}{l}\text { Razón de } \\
\text { Verosimilitudes }\end{array}$ & 8.769 & 6 & 0.187 \\
\hline & Siempre & 0 & 1 & 0 & 0 & 1 & $\begin{array}{l}\text { Asociación } \\
\text { lineal por lineal }\end{array}$ & 2.2 & \begin{tabular}{|lll}
1 & &
\end{tabular} & 0.138 \\
\hline & Total & 5 & 4 & 2 & 9 & 20 & $\begin{array}{l}\mathrm{N} \text { de casos } \\
\text { validos }\end{array}$ & 20 & & \\
\hline
\end{tabular}

Fuente: Elaboración propia.

Ahora bien, el 100\% han estado estresados en algún momento, esto se refleja en los 20 colaboradores al relacionarlo con el ruido se aprecia que 11 colaboradores en un 55\% expresaron que siempre y casi siempre hay demasiado ruido en el área donde desarrollan su trabajo. Al aplicar la técnica de chi cuadrado a ambas variables dio como resultado una significancia asíntota bilateral de 0.247. Es importante resaltar que, al preguntársele al responsable de recursos humanos, si consideraba que las condiciones laborales pueden ser un desencadenante del estrés, argumento que sí, porque cuando no se cuenta con las condiciones que se requiere, genera más tensión, el no tener un espacio suficiente en la oficina, una buena iluminación y ventilación. Con la aplicación del Chi cuadrado de Pearson y el resultado de cada asíntota bilateral mayor a 0.05 , respalda la aceptación de la hipótesis nula, reafirmando que las condiciones laborales no influyen en el estrés laboral de los colaboradores del Centro de Salud "Adán Barillas Huete".

\section{Discusión y conclusiones}

De acuerdo con Agosto (2008), el estrés es un estado patológico crónico ocasionado por las condiciones habituales de trabajo, entonces se puede afirmar que, en el centro de salud, los colaboradores en un 100\% han presentado esta patología.

Reafirmando lo planteado por Rodríguez, Molerio \& Roque que el estrés aparece por la intensidad de la demanda laboral o por problemas de índoles organizacionales, por ende, el 70\% de los colaborares están presionados por su jefe, desarrollando sus actividades bajo presión. Además, en un 80\% tienen un alto grado de responsabilidad en el cargo que ocupan, tanto el personal administrativo, medico y enfermeros. 
Aunque en el Código del Trabajo de Nicaragua en su arto. 50 establece, el día natural para efectos del trabajo es el comprendido entre las 6 de la mañana y las ocho de la noche, los colaboradores en un $90 \%$ cumplen jornadas labores muy extensas en relación a los horarios establecidos en dicho código.

De acuerdo con Mutua Colaboradora con la Seguridad Social que los efectos del estrés son de índole fisiológica, psicológica y conductual, y apoyado en los resultado del estudio de Borjan (2016) donde concluye que el dolor de cabeza fue uno de los efectos, es apremiante destacar que los colaboradores del centro de salud han presentado en un $95 \%$, efectos de orden físico, reflejados en constantes jaquecas.

Tomando en consideración lo expresado por Chiavenato (2007) que las condiciones ambientales del trabajo están referidas a las circunstancias físicas que rodean al empleado. Es importante enunciar que en un 90\% las instalaciones del centro de salud facilitan a los colaboradores el desarrollo de su trabajo. En un $65 \%$ se realiza limpieza en cada una de las áreas de trabajo y en un 55\% la iluminación es adecuada, además el mismo autor señala que el ruido no provoca disminución en el desempeño del trabajo, aunque el $55 \%$ de los colaboradores expresaron que hay constante ruido sin haberse determinado el nivel de decibeles.

Lógicamente, que al relacionar las condiciones laborales con la implicancia en el estrés laboral, aplicando la técnica de Chi Cuadrado de Pearson, se logro constatar que las condiciones laborales no tienen implicancia con el estrés laboral ya que sus resultados de significancias asíntota bilateral expresaron mayor a 0.05 , dando lugar a aceptar la hipótesis nula, las condiciones laborales no influyen en el estrés laboral de los colaboradores del centro de salud Adán Barrillas Huete.

Finalmente, se infiere que la alta demanda de servicios de salud, acompañado de las exigencias en el cumplimiento de las metas, podrían ser los causantes del estrés laboral de los colaboradores en esta organización, sustentado en el estudio realizado por Alaniz (2006) donde concluyo que los factores del estrés laboral fueron la carga laboral expresado en volumen de pacientes.

\section{Agradecimiento}

Se agradece a la Dra. Karla Rothschuh Pérez, Directora del Centro de Salud Adán Barillas Huete por permitir desarrollar el estudio y a los colaboradores por haber proporcionado información veraz y oportuna, lográndose ejecutar extensión universitaria en temáticas vigentes y en el ámbito local. 


\section{Lista de referencias}

Acosta, J. M. (2008). Gestión del Estres. Barcelona: Bresca Editorial, S.L.

Alaniz, T. R. (Junio de 2016). Estrés Laboral en Médicos Residentes de los Hopitales pùblicos de Managua. Managua, Nicaragua.

Asamblea Nacional De la República de Nicaragua. (1996). Codigo del trabajo. Obtenido de legislacion.asamblea.gob.ni

Bernal, C. A. (2006). Metodologia de la investigación, 2da edicion. Mexico: Pearson Educacion.

Borjan, S. E. (2016). Estres laboral en personal de enfermeria en la unidad de cuidados intensivos pediatricos.

Chiavenato, I. (2007). Administracion de Recursos Humanos.

Feldman, R. S. (2006). Psicologia co aplicaciones en paises de habla Hispana. MC Graw Hill.

Malhotra, N. k. (2008). Investigacion de mercados. Mexico: Pearson Educación.

Mutua MC mutual colaboradora con la Seguridad Social. (2008). Stop al estrés: Como gestionar el Estrés Laboral. Barcelona: MC mutual.

Robbins, S. P. (2004). Comportamiento Organizacional, 10a. ed. Mexico: Pearson Educacion.

Rodriguez, R., Osana Molerio, \& Roque, Y. (Enero de 2002). Revista internacional de Psicologia. Obtenido de www.revistapsicologia.org

Soto, C., \& Lautenschlager, G. (2003). Comparacion estadistica de la confiabilidad Alfa de Cronbach: aplicaciones en la medicion educacional y psicologica. Revista de Psicologia. Obtenido de www.redalyc.org

Tamayo, M. T. (2008). Diccionario de la investigación cientifica, 2da edición. Mexico: Limusa.

Union General de trabajadores UGT. (sf). Prevencion de riesgos laborales. UGT.

Whetten, D., Kim, \& Cameron, K. (2011). Desarrollo de habilidades directivas, 8 edicion. Mexico: Pearson Educacion. 